\title{
Numerical Analysis of Arbitrarily Shaped Probe-Excited Single-Arm Printed Wire Antennas
}

\author{
Rong-Lin Li and Hisamatsu Nakano, Fellow, IEEE
}

\begin{abstract}
A general integral equation technique is described for analysis of an arbitrarily shaped single-arm printed wire antenna excited through a vertical probe. A unified current integral equation is formulated on the basis of dyadic Green's functions and the reciprocity theorem. The current distribution is obtained by using a parametric moment method in which parameter segments are adopted for the printed wire instead of the commonly employed wire length segments. The radiation field solution involving both the printed antenna and vertical probe is also presented. The validity of the formulation is verified by comparing the numerically obtained input impedance and radiation patterns for a linear antenna and a meander antenna with measured data. A circular open loop and an Archimedian spiral are investigated to illustrate the applicability of the present technique.
\end{abstract}

Index Terms - Integral equation, microstrip antennas, moment method, printed antennas, probe feed.

\section{INTRODUCTION}

$\mathbf{F}$ IOR more than ten years, printed wire antennas have been numerically investigated by a number of researchers [1]-[7]. In all of these works, it is assumed that the antennas are excited by a center-fed delta-gap voltage source. In order to realize such a center-fed antenna, a balun circuit [8] must be used, which increases the complexity of the system.

The coaxial feed has a relatively simple structure. Using a coaxial feed, a printed antenna (horizontal element) is excited through a vertical probe, which is formed by extending the inner conductor of the coaxial line. The numerical techniques presented by the above researchers, unfortunately, are incapable of handling such a vertical coaxial feed model since the formulations include only the effects of the horizontal element.

Recently, a numerical analysis was presented in [9] for single-arm printed antennas excited through a vertical feed wire. The numerical model in this work, however, was developed only for an Achimedian spiral. The feeding model has not been discussed in any detail.

Our intention in this paper is to present a general numerical analysis for a probe-excited single-arm printed wire antenna with an arbitrary shape. The analysis is based on an integral equation approach.

First, a unified current integral equation governing the current distribution along the vertical probe and printed wire

Manuscript received December 31, 1997; revised June 16, 1998.

R.-L. Li is with the Department of Electrical Engineering, Zhejiang University, Hangzhou, 310027 China.

H. Nakano is with the College of Engineering, Hosei University, Koganei, Tokyo 184, Japan.

Publisher Item Identifier S 0018-926X(98)06873-2.

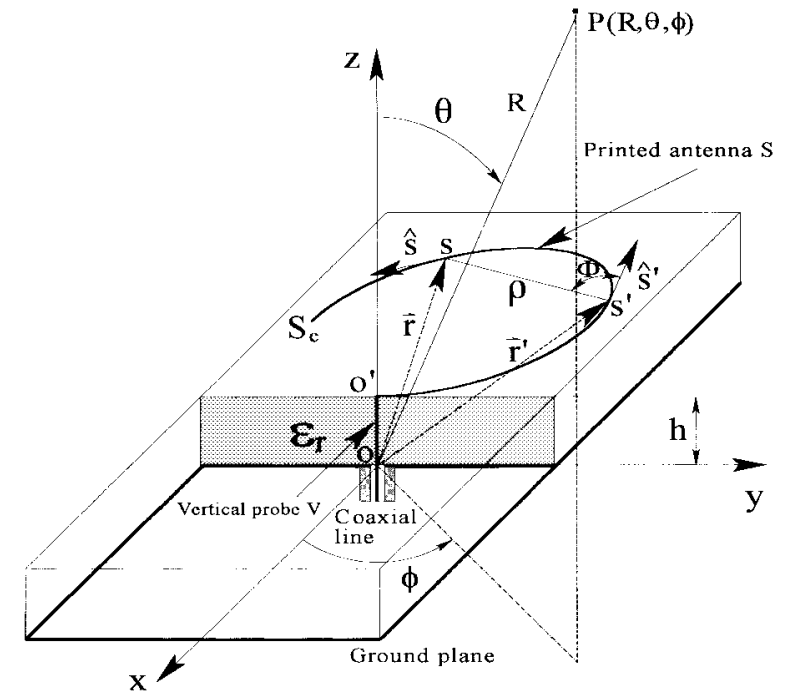

Fig. 1. Geometry of an arbitrarily shaped single-arm printed wire antenna excited by a vertical probe.

antenna is formulated. This equation is then solved using the moment method based on a parametric representation of the printed wire geometry. A concise computational formulation for the far-zone fields radiated from the vertical probe and printed antenna is also presented. To verify the validity of the formulation, the numerical results of the input impedance and radiation patterns for a linear antenna and a meander antenna are compared with measured data. Finally, a circular open loop and an Archimedian spiral are investigated to demonstrate the applicability of the present approach.

\section{FORMULATION}

\section{A. Current Integral Equation}

The probe-excited single-arm printed wire antenna is illustrated in Fig. 1. An arbitrarily shaped thin wire antenna $S$ with wire radius $a_{s}$ is printed on a grounded dielectric substrate with thickness $h$ and relative permittivity $\varepsilon_{r}$. The printed antenna is excited at the starting point $o^{\prime}$ of the printed wire through a vertical probe $V$. The inner and outer conductor radii of the coaxial line are $a_{v}$ and $b$, respectively. It is assumed that a source point (whose position vector is $\vec{r}^{\prime}$ ) is situated on the wire axis and an observation point (whose position vector is $\vec{r}$ ) is located on the wire surface with a thin-wire approximation (that is, both $a_{v}$ and $a_{s}$ are much less than the free-space wavelength $\lambda_{0}$ ). The electric field at the observation point is 
expressed as

$$
\begin{gathered}
\vec{E}(\vec{r})=\int_{L}\left(k^{2}(\vec{r}) \overline{\bar{U}}+\nabla \nabla\right) \cdot \overline{\bar{G}}\left(\vec{r}, \vec{r}^{\prime}\right) \cdot \hat{l}^{\prime} I\left(l^{\prime}\right) d l^{\prime}, \\
L=V+S
\end{gathered}
$$

where $I\left(l^{\prime}\right)$ is the unknown total axial current, $\overline{\bar{U}}$ is the unit dyadic, $\overline{\bar{G}}\left(\vec{r}, \vec{r}^{\prime}\right)$ is the potential vector dyadic Green's function, and

$$
k^{2}(\vec{r})= \begin{cases}\varepsilon_{r} k_{0}^{2}, & \vec{r} \in V \\ k_{0}^{2}, & \vec{r} \in S\end{cases}
$$

with $k_{0}=2 \pi / \lambda_{0}$.

Therefore, the tangential component of the electric field along the wire is

$$
E_{l}(\vec{r})= \begin{cases}\int_{V}\left(\varepsilon_{r} k_{0}^{2} \hat{z} \cdot \overline{\bar{U}}+\hat{z} \cdot \nabla \nabla\right) \cdot \overline{\bar{G}^{z}}\left(\vec{r}, \vec{r}^{\prime}\right) & \\ \cdot \hat{z}^{\prime} I\left(z^{\prime}\right) d z^{\prime}+\int_{S}\left(\varepsilon_{r} k_{0}^{2} \hat{z} \cdot \overline{\bar{U}}+\hat{z} \cdot \nabla \nabla\right) & \\ \cdot \overline{\bar{G}}^{s}\left(\vec{r}, \vec{r}^{\prime}\right) \cdot \hat{s}^{\prime} I\left(s^{\prime}\right) d s^{\prime}, & \vec{r} \in V \\ \int_{V}\left(k_{0}^{2} \hat{s} \cdot \overline{\bar{U}}+\hat{s} \cdot \nabla \nabla\right) \cdot \overline{\bar{G}}^{z}\left(\vec{r}, \vec{r}^{\prime}\right) & \\ \cdot \hat{z}^{\prime} I\left(z^{\prime}\right) d z^{\prime}+\int_{S}\left(k_{0}^{2} \hat{s} \cdot \overline{\bar{U}}+\hat{s} \cdot \nabla \nabla\right) & \\ \cdot \overline{\bar{G}}^{s}\left(\vec{r}, \vec{r}^{\prime}\right) \cdot \hat{s}^{\prime} I\left(s^{\prime}\right) d s^{\prime}, & \vec{r} \in S\end{cases}
$$

where $\overline{\bar{G}}^{z}\left(\vec{r}, \vec{r}^{\prime}\right)$ and $\overline{\bar{G}} s\left(\vec{r}, \vec{r}^{\prime}\right)$ are the dyadic Green's functions for a $\hat{z}^{\prime}$-oriented vertical electric dipole (VED) embedded in the dielectric substrate and an $\hat{s}^{\prime}$-oriented horizontal electric dipole (HED) on the dielectric substrate, respectively. by

The dyadic Green's function of the $\hat{z}^{\prime}$-oriented VED is given

$$
\overline{\bar{G}} z\left(\vec{r}, \vec{r}^{\prime}\right)= \begin{cases}G_{V}^{z}\left(z, z^{\prime}\right) \hat{z}^{\prime} \hat{z}^{\prime}, & \vec{r} \in V \\ G_{S}^{z}\left(\rho, z^{\prime}\right) \hat{z}^{\prime} \hat{z}^{\prime}, & \vec{r} \in S\end{cases}
$$

where $G_{V}^{z}\left(z, z^{\prime}\right)$ and $G_{S}^{z}\left(\rho, z^{\prime}\right)$ are represented by Sommerfeld integrals [10] $\left(e^{j \omega t}\right.$ time dependence is assumed and omitted throughout this paper)

$$
\begin{aligned}
G_{V}^{z}\left(z, z^{\prime}\right)= & -\frac{60 j}{\varepsilon_{r} k_{0}} \\
& \left\{\begin{array}{l}
\int_{0}^{\infty} \frac{\lambda}{u f_{\mathrm{TM}}} \cosh \left(u z^{\prime}\right)[u \cosh [u(h-z)] \\
\left.+\varepsilon_{r} u_{0} \sinh [u(h-z)]\right] \\
\cdot J_{0}\left(\lambda a_{v}\right) d \lambda, \quad z^{\prime} \leq z \leq h \\
\int_{0}^{\infty} \frac{\lambda}{u f_{\mathrm{TM}}} \cosh (u z)\left[u \cosh \left[u\left(h-z^{\prime}\right)\right]\right. \\
\left.+\varepsilon_{r} u_{0} \sinh \left[u\left(h-z^{\prime}\right)\right]\right] \\
\cdot J_{0}\left(\lambda a_{v}\right) d \lambda, \quad 0 \leq z \leq z^{\prime} \\
60,
\end{array}\right.
\end{aligned}
$$$$
G_{S}^{z}\left(\rho, z^{\prime}\right)=\lim _{z \rightarrow h}-\frac{60 j}{k_{0}} \int_{0}^{\infty} \frac{\lambda}{f_{\mathrm{TM}}}
$$$$
\cdot \cosh \left(u z^{\prime}\right) e^{-u_{0}(z-h)} J_{0}(\lambda \rho) d \lambda \text {. }
$$

$\overline{\bar{G}}^{s}\left(\vec{r}, \vec{r}^{\prime}\right)$ for the $\hat{s}^{\prime}$-oriented HED is [11]

$$
\begin{aligned}
\overline{\bar{G}}^{s} & \left(\vec{r}, \bar{r}^{\prime}\right) \\
& = \begin{cases}G_{s, V}^{s}\left(z, \rho_{v}\right) \hat{s}^{\prime} \hat{s}^{\prime}+G_{z, V}^{s}\left(z, \rho_{v}\right) \hat{z}^{\prime} \hat{s}^{\prime}, & \vec{r} \in V \\
G_{s, S}^{s}(\rho) \hat{s}^{\prime} \hat{s}^{\prime}+G_{z, S}^{s}(\rho) \hat{z}^{\prime} \hat{s}^{\prime}, & \vec{r} \in S\end{cases}
\end{aligned}
$$

where

$$
\begin{aligned}
G_{s, V}^{s}\left(z, \rho_{v}\right)= & -\frac{60 j}{\varepsilon_{r} k_{0}} \int_{0}^{\infty} \frac{\sinh (u z)}{f_{\mathrm{TE}}} \cdot J_{0}\left(\lambda \rho_{v}\right) \lambda d \lambda \\
G_{z, V}^{s}\left(z, \rho_{v}\right)= & -\frac{60 j}{\varepsilon_{r} k_{0}}\left(1-\varepsilon_{r}\right) \cos \Phi \int_{0}^{\infty} \\
& \cdot \frac{\sinh (u h) \cosh (u z)}{f_{\mathrm{TM}} f_{\mathrm{TE}}} \cdot J_{1}\left(\lambda \rho_{v}\right) \lambda^{2} d \lambda \\
G_{s, S}^{s}(\rho)= & \lim _{z \rightarrow h}-\frac{30 j}{k_{0}}\left\{\frac{e^{-j k_{0} R_{z}}}{R_{z}}+\int_{0}^{\infty} \frac{\lambda}{u_{0} f_{\mathrm{TE}}}\right. \\
& \cdot\left[u_{0} \sinh (u h)-u \cosh (u h)\right] \\
& \left.\cdot e^{-u_{0}(z-h)} \cdot J_{0}(\lambda \rho) d \lambda\right\} \\
G_{z, S}^{s}(\rho)= & \lim _{z \rightarrow h}-\frac{60 j}{k_{0}}\left(1-\varepsilon_{r}\right) \cos \Phi \int_{0}^{\infty} \\
& \cdot \frac{\sinh (u h) \cosh (u h)}{f_{\mathrm{TM}} f_{\mathrm{TE}}} \\
& \cdot e^{-u_{0}(z-h)} \cdot J_{1}(\lambda \rho) \lambda^{2} d \lambda
\end{aligned}
$$

In (5)-(11), $J_{n}(\bullet)$ represents the $n$th order Bessel function of the first kind

$$
\begin{aligned}
u & =\sqrt{\lambda^{2}-\varepsilon_{r} k_{0}^{2}}, \quad u_{0}=\sqrt{\lambda^{2}-k_{0}^{2}} \\
f_{\mathrm{TE}} & =u_{0} \sinh (u h)+u \cosh (u h) \\
f_{\mathrm{TM}} & =\varepsilon_{r} u_{0} \cosh (u h)+u \sinh (u h) \\
R_{z} & =\sqrt{\rho^{2}+(h-z)^{2}}
\end{aligned}
$$

and

$$
\begin{aligned}
\rho_{v} & =\sqrt{\left(x-x^{\prime}\right)^{2}+\left(y-y^{\prime}\right)^{2}+a_{v}^{2}} \\
\rho & =\sqrt{\left(x-x^{\prime}\right)^{2}+\left(y-y^{\prime}\right)^{2}+a_{s}^{2}}
\end{aligned}
$$

where $(x, y)$ and $\left(x^{\prime}, y^{\prime}\right)$ are, respectively, the Cartesian coordinates of the observation and source points along the wire axis. Substituting (4) and (7) into (3) and letting $\nabla=$ $\nabla_{t}+\hat{z}(\partial / \partial z)$, where the subscript $t$ indicates transverse to the $z$ component of the operator $\nabla$, we have

$$
E_{l}(\vec{r})=\left\{\begin{aligned}
\int_{S} & \left\{\varepsilon_{r} k_{0}^{2} G_{z, V}^{s}\left(z, \rho_{v}\right)+\frac{\partial}{\partial z}\right. \\
\cdot & {\left[\nabla_{t} G_{s, V}^{s}\left(z, \rho_{v}\right) \cdot \hat{s}^{\prime}\right.} \\
& \left.\left.+\frac{\partial}{\partial z} G_{z, V}^{s}\left(z, \rho_{v}\right)\right]\right\} I\left(s^{\prime}\right) d s^{\prime} \\
& +\int\left\{\varepsilon_{r} k_{0}^{2} G_{V}^{z}\left(z, z^{\prime}\right)+\frac{\partial^{2}}{\partial z^{2}}\left[G_{V}^{z}\left(z, z^{\prime}\right)\right]\right\} \\
\cdot & I\left(z^{\prime}\right) d z^{\prime}, \\
\int_{S} & \left\{k_{0}^{2} G_{s, S}^{s}(\rho)\left[\hat{s} \cdot \hat{s}^{\prime}\right]+\frac{\partial}{\partial s}\right. \\
& \left.\cdot\left[\nabla_{t} G_{s, S}^{s}(\rho) \cdot \hat{s}^{\prime}+\frac{\partial}{\partial z} G_{z, S}^{s}(\rho)\right]\right\} \\
& +\int_{V} \frac{\partial^{2}}{\partial s \partial z}\left[G_{S}^{z}\left(\rho, z^{\prime}\right)\right] I\left(z^{\prime}\right) d z^{\prime},
\end{aligned}\right.
$$

It is noted from (8) and (10) that

$$
\begin{gathered}
\nabla_{t} G_{s, V}^{s}\left(z, \rho_{v}\right)=-\nabla_{t}^{\prime} G_{s, V}^{s}\left(z, \rho_{v}\right) \\
\nabla_{t} G_{s, S}^{s}(\rho)=-\nabla_{t}^{\prime} G_{s, S}^{s}(\rho) .
\end{gathered}
$$


Substitution of (15) and (16) into (14) leads to

$$
\begin{aligned}
& \left\{\begin{array}{c}
\int_{S}\left\{\varepsilon_{r} k_{0}^{2} G_{z, V}^{s}\left(z, \rho_{v}\right)+\frac{\partial}{\partial z}\right. \\
\cdot\left[-\frac{\partial}{\partial s^{\prime}} G_{s, V}^{s}\left(z, \rho_{v}\right)\right.
\end{array}\right. \\
& \left.\left.+\frac{\partial}{\partial z} G_{z, V}^{s}\left(z, \rho_{v}\right)\right]\right\} I\left(s^{\prime}\right) d s^{\prime} \\
& E_{l}(\vec{r})=\left\{\begin{array}{c}
\quad \int_{V}\left\{\varepsilon_{r} k_{0}^{2} G_{V}^{z}\left(z, z^{\prime}\right)+\frac{\partial^{2}}{\partial z^{2}}\left[G_{V}^{z}\left(z, z^{\prime}\right)\right]\right\} \\
\cdot I\left(z^{\prime}\right) d z^{\prime}, \\
\int_{S}\left\{k_{0}^{2} G_{s, S}^{s}(\rho)\left[\hat{s} \cdot \hat{s}^{\prime}\right]+\frac{\partial}{\partial s}\right. \\
\left.\cdot\left[-\frac{\partial}{\partial s^{\prime}} G_{s, S}^{s}(\rho)+\frac{\partial}{\partial z} G_{z, S}^{s}(\rho)\right]\right\} I\left(s^{\prime}\right) d s^{\prime} \\
+\int_{V} \frac{\partial^{2}}{\partial s \partial z}\left[G_{S}^{z}\left(\rho, z^{\prime}\right)\right] I\left(z^{\prime}\right) d z^{\prime}, \quad \vec{r} \in S .
\end{array}\right.
\end{aligned}
$$

Using

$$
J_{1}(\lambda \rho) \lambda=-\frac{d J_{0}(\lambda \rho)}{d \rho}
$$

and

$$
\cos \Phi=-\frac{\partial \rho}{\partial s^{\prime}} \quad(\text { see Appendix })
$$

we have

$$
\begin{aligned}
\frac{\partial}{\partial z} G_{z, V}^{s}\left(z, \rho_{v}\right) & =-\frac{\partial}{\partial s^{\prime}} \tilde{G}_{z, V}^{s}\left(z, \rho_{v}\right) \\
\frac{\partial}{\partial z} G_{V}^{z}\left(z, z^{\prime}\right) & =-\frac{\partial}{\partial z^{\prime}} \tilde{G}_{V}^{z}\left(z, z^{\prime}\right) \\
\frac{\partial}{\partial z} G_{z, S}^{s}(\rho) & =-\frac{\partial}{\partial s^{\prime}} \tilde{G}_{z, S}^{s}(\rho) \\
\frac{\partial}{\partial z} G_{S}^{z}\left(\rho, z^{\prime}\right) & =-\frac{\partial}{\partial z^{\prime}} \tilde{G}_{S}^{z}\left(\rho, z^{\prime}\right)
\end{aligned}
$$

where

$$
\begin{aligned}
& \tilde{G}_{z, V}^{s}\left(z, \rho_{v}\right) \\
& =-\frac{60 j}{\varepsilon_{r} k_{0}}\left(\varepsilon_{r}-1\right) \int_{0}^{\infty} \frac{u \sinh (u h) \sinh (u z)}{f_{\mathrm{TM}} f_{\mathrm{TE}}} \\
& \quad \cdot J_{0}\left(\lambda \rho_{v}\right) \lambda d \lambda \\
& \tilde{G}_{V}^{z}\left(z, z^{\prime}\right) \\
& =-\frac{60 j}{\varepsilon_{r} k_{0}} \\
& \quad \begin{array}{c}
\int_{0}^{\infty} \frac{\lambda}{u f_{\mathrm{TM}}} \sinh \left(u z^{\prime}\right)[u \sinh [u(h-z)] \\
\left.+\varepsilon_{r} u_{0} \cosh [u(h-z)]\right] \\
\cdot J_{0}\left(\lambda a_{v}\right) d \lambda, \quad z^{\prime} \leq z \leq h \\
\int_{0}^{\infty} \frac{\lambda}{u f_{\mathrm{TM}}} \sinh (u z)\left[u \sinh \left[u\left(h-z^{\prime}\right)\right]\right. \\
\left.+\varepsilon_{r} u_{0} \cosh \left[u\left(h-z^{\prime}\right)\right]\right] \\
\cdot J_{0}\left(\lambda a_{v}\right) d \lambda, \quad 0 \leq z \leq z^{\prime}
\end{array} \\
& \tilde{G}_{z, S}^{s}(\rho) \quad \begin{array}{c}
60 j \\
=
\end{array} \\
& \quad \cdot J_{0}(\lambda \rho) \lambda d \lambda \\
& k_{0}\left(1-\varepsilon_{r}\right) \int_{0}^{\infty} \frac{u_{0} \sinh (u h) \cosh (u h)}{f_{\mathrm{TM}} f_{\mathrm{TE}}}
\end{aligned}
$$

$$
\begin{aligned}
& \tilde{G}_{S}^{z}\left(\rho, z^{\prime}\right) \\
& \quad=-\frac{60 j}{k_{0}} \int_{0}^{\infty} \frac{u_{0} \lambda}{u f_{\mathrm{TM}}} \sinh \left(u z^{\prime}\right) J_{0}(\lambda \rho) d \lambda .
\end{aligned}
$$

Note that the Sommerfeld integrals in (24)-(27) involve only the Bessel function of the first kind and zero order.

Inserting (20)-(23) into (17) yields

$$
E_{l}(\vec{r})=\left\{\begin{array}{l}
\int_{S}\left\{\varepsilon_{r} k_{0}^{2} G_{z, V}^{s}\left(z, \rho_{v}\right)-\frac{\partial^{2}}{\partial s^{\prime} \partial z}\right. \\
\left.\cdot\left[G_{s, V}^{s}\left(z, \rho_{v}\right)+\tilde{G}_{z, V}^{s}\left(z, \rho_{v}\right)\right]\right\} I\left(s^{\prime}\right) d s^{\prime} \\
+\int_{V}\left\{\varepsilon_{r} k_{0}^{2} G_{V}^{z}\left(z, z^{\prime}\right)-\frac{\partial^{2}}{\partial z^{\prime} \partial z}\right. \\
\left.\cdot\left[\tilde{G}_{V}^{z}\left(z, z^{\prime}\right)\right]\right\} I\left(z^{\prime}\right) d z^{\prime}, \\
\int_{S}\left\{k_{0}^{2} G_{s, S}^{s}(\rho)\left[\hat{s} \cdot \hat{s}^{\prime}\right]-\frac{\partial^{2}}{\partial s^{\prime} \partial s} \in V\right. \\
\left.\cdot\left[G_{s, S}^{s}(\rho)+\tilde{G}_{z, S}^{s}(\rho)\right]\right\} I\left(s^{\prime}\right) d s^{\prime} \\
-\int_{V} \frac{\partial^{2}}{\partial z^{\prime} \partial s}\left[\tilde{G}_{S}^{z}\left(\rho, z^{\prime}\right)\right] I\left(z^{\prime}\right) d z^{\prime},
\end{array} \quad \vec{r} \in S .\right.
$$

It is worth mentioning that the integrand in the first right-hand term of (28) is simplified as

$$
\begin{aligned}
& \left.\varepsilon_{r} k_{0}^{2} G_{z, V}^{s}\left(z, \rho_{v}\right)-\frac{\partial^{2}}{\partial s^{\prime} \partial z}\left[G_{s, V}^{s}\left(z, \rho_{v}\right)\right]+\tilde{G}_{z, V}^{s}\left(z, \rho_{v}\right)\right] \\
& =-\frac{\partial^{2}}{\partial s^{\prime} \partial z}\left[\tilde{G}_{S}^{z}\left(\rho_{v}, z\right)\right] .
\end{aligned}
$$

Hence, substituting (29) into (28) and integrating by parts, we obtain

$$
E_{l}(\vec{r})=\left\{\begin{aligned}
\int_{S} & \frac{d I\left(s^{\prime}\right)}{d s^{\prime}} \frac{\partial}{\partial z}\left[\tilde{G}_{S}^{z}\left(\rho_{v}, z\right)\right] d s^{\prime} \\
& -\left\{I\left(s^{\prime}\right) \frac{\partial}{\partial z}\left[\tilde{G}_{S}^{z}\left(\rho_{v}, z\right)\right]\right\}_{s^{\prime}=o^{\prime}}^{s^{\prime}=S_{e}} \\
& +\int_{V}\left\{\varepsilon_{r} k_{0}^{2} G_{V}^{z}\left(z, z^{\prime}\right) I\left(z^{\prime}\right)\right. \\
& \left.+\frac{d I\left(z^{\prime}\right)}{d z^{\prime}} \frac{\partial}{\partial z}\left[\tilde{G}_{V}^{z}\left(z, z^{\prime}\right)\right]\right\} d z^{\prime} \\
& -\left\{I\left(z^{\prime}\right) \frac{\partial}{\partial z}\left[\tilde{G}_{V}^{z}\left(z, z^{\prime}\right)\right]\right\}_{z^{\prime}=0}^{z^{\prime}=h}, \vec{r} \in V \\
\int_{S} & \left\{k_{0}^{2} G_{s, S}^{s}(\rho)\left[\hat{s}^{\prime}, \hat{s}^{\prime}\right] I\left(s^{\prime}\right)\right. \\
& \left.+\frac{d I\left(s^{\prime}\right)}{d s^{\prime}} \frac{\partial}{\partial s}\left[G_{s, S}^{s}(\rho)+\tilde{G}_{z, S}^{s}(\rho)\right]\right\} d s^{\prime} \\
& -\left\{I\left(s^{\prime}\right) \frac{\partial}{\partial s}\left[G_{s, S}^{s}(\rho)+\tilde{G}_{z, S}^{s}(\rho)\right]\right\}_{s^{\prime}=S_{e}^{\prime}} \\
& +\int_{V} \frac{d I\left(z^{\prime}\right)}{d z^{\prime}} \frac{\partial}{\partial s}\left[\tilde{G}_{S}^{z}\left(\rho, z^{\prime}\right)\right] d z^{\prime} \\
& -\left\{I\left(z^{\prime}\right) \frac{\partial}{\partial s}\left[\tilde{G}_{S}^{z}\left(\rho, z^{\prime}\right)\right]\right\}_{z^{\prime}=0}^{z^{\prime}=h}, \vec{r} \in S
\end{aligned}\right.
$$

where $S_{e}$ indicates the arm-end point at the printed wire. 
From (25) and (27), it is evident that

$$
\begin{aligned}
& \left.\tilde{G}_{V}^{z}\left(z, z^{\prime}\right)\right|_{z^{\prime}=0}=0 \\
& \left.\tilde{G}_{V}^{z}\left(z, z^{\prime}\right)\right|_{z^{\prime}=h}=\left.\tilde{G}_{S}^{z}\left(\rho_{v}, z\right)\right|_{s^{\prime}=o^{\prime}}
\end{aligned}
$$

By using (31) and (32) and the current boundary condition at the arm-end point $S_{e}$, i.e., $I\left(S_{e}\right)=0$, the final expression for $E_{t}(\vec{r})$ is

$$
E_{l}(\vec{r})=\left\{\begin{aligned}
\int_{S} & \frac{d I\left(s^{\prime}\right)}{d s^{\prime}} \frac{\partial}{\partial z}\left[\tilde{G}_{S}^{z}\left(\rho_{v}, z\right)\right] d s^{\prime} \\
& +\int_{V}\left\{\varepsilon_{r} k_{0}^{2} G_{V}^{z}\left(z, z^{\prime}\right) I\left(z^{\prime}\right)\right. \\
& \left.+\frac{d I\left(z^{\prime}\right)}{d z^{\prime}} \frac{\partial}{\partial z}\left[\tilde{G}_{V}^{z}\left(z, z^{\prime}\right)\right]\right\} d z^{\prime}, \quad \vec{r} \in V \\
\int_{S} & \left\{k_{0}^{2} G_{s, S}^{s}(\rho)\left[\hat{s} \cdot \hat{s}^{\prime}\right] I\left(s^{\prime}\right)\right. \\
& \left.+\frac{d I\left(s^{\prime}\right)}{d s^{\prime}} \frac{\partial}{\partial s}\left[G_{s, S}^{s}(\rho)+\tilde{G}_{z, S}^{s}(\rho)\right]\right\} d s^{\prime} \\
& +\int_{V} \frac{d I\left(z^{\prime}\right)}{d z^{\prime}} \frac{\partial}{\partial s}\left[\tilde{G}_{S}^{z}\left(\rho, z^{\prime}\right)\right] d z^{\prime} \\
& -I(h) \frac{\partial}{\partial s}\left\{\tilde{G}_{S}^{z}(\rho, h)\right. \\
& \left.-\left[G_{s, S}^{s}(\rho)+\tilde{G}_{z, S}^{s}(\rho)\right]\right\}\left.\right|_{s^{\prime}=o^{\prime}}, \quad \vec{r} \in S .
\end{aligned}\right.
$$

Note that (33) involves only the first-order derivative of Sommerfeld integrals. This derivative is removed by virtue of integration by parts when the impedance matrix elements, which will be shown later in (45) and (46), are calculated.

The reciprocity theorem is used to develop the current integral equation. Under the thin-wire approximation assumption, the reaction current integral equation is expressed as [10]

$$
\int_{L} E_{l}^{T}(\vec{r}) I(l) d l=\int_{0}^{h} \phi_{V}\left(z^{\prime}\right) T_{C}\left(z^{\prime}\right) d z^{\prime}
$$

where $T_{C}$ denotes the test current source, $E_{l}^{T}(\vec{r})$ is the tangential electric field at the observation point $(\vec{r})$ due to the test current source $T_{C}\left(l^{\prime}\right)\left(l^{\prime} \in L\right) . \phi_{V}$ is the reaction quantity of the magnetic current distribution and the magnetic field generated by a VED at $z=z^{\prime}$ over the coaxial aperture, which is given by [10]

$$
\begin{aligned}
\phi_{V}\left(z^{\prime}\right)= & \frac{V_{0}}{\ln \left(b / a_{v}\right)} \int_{0}^{\infty} \frac{\lambda}{u}\left[\frac{R_{\mathrm{TM}} e^{-u z^{\prime}}+e^{-u\left(2 h-z^{\prime}\right)}}{D_{\mathrm{TM}}}\right] \\
& \cdot\left[J_{0}(\lambda b)-J_{0}\left(\lambda a_{v}\right)\right] d \lambda
\end{aligned}
$$

where $V_{0}(=1 \mathrm{~V})$ is the voltage applied and

$$
R_{\mathrm{TM}}=\frac{u+\varepsilon_{r} u_{0}}{u-\varepsilon_{r} u_{0}}, \quad D_{\mathrm{TM}}=R_{\mathrm{TM}}-e^{-2 u h} .
$$

\section{B. Parametric MoM Solution}

The current integral equation in (34) can be solved using the method of moments (MoM). In this section, a parametric MoM is used, which is more suitable to curved wire antennas [12]. The parametric MoM has two important features: 1) the geometry of the wire is represented with a general parametric equation rather than the commonly used piecewise length segments and 2) the basis function is directly defined as a function of the parametric variable instead of the wire length.

Without loss of generality, we begin with representing the geometry of the printed wire axis in a parametric form

$$
\vec{r}(t)=x(t) \hat{x}+y(t) \hat{y}+h \hat{z} \quad T_{0} \leq t \leq T \quad \vec{r} \in S
$$

where $\bar{r}\left(T_{0}\right)$ and $\bar{r}(T)$ corresponds to the position vectors for points $o^{\prime}$ and $S_{e}$, respectively. Note that the parametric variable $t$ varies from $T_{0}$ to $T$ continuously. For convenience, we choose the parameter $T_{0}$ numerically as $T_{0}=h$.

Dividing the interval $[0, h]$ of the thickness into $\left(z_{0}=\right.$ $\left.0, z_{1}, \cdots, z_{N_{V}}=h\right)\left(N_{V}\right.$ is the number of length segments over the vertical probe) and the interval $[h, T]$ of the parameter $t$ into $\left(t_{N_{V}}=h, t_{N_{V}+1}, \cdots, t_{N_{V}+N_{S}}=T\right)\left(N_{S}\right.$ is the number of parameter segments over the horizontal printed antenna), we express the unknown current distribution $I(l)$ as

$$
I(l) \cong\left\{\begin{array}{lll}
I_{V}(z)=\sum_{n=0}^{N_{V}} I_{n} B_{n}(z) & (0 \leq z \leq h) & l \in V \\
I_{S}(t)=\sum_{n=N_{V}}^{N_{V}+N_{S}} I_{n} B_{n}(t) & (h \leq t \leq T) \quad l \in S
\end{array}\right.
$$

where $I_{n}$ 's are unknown current coefficients. $B_{n}(z)(n=$ $\left.0,1, \cdots, N_{V}\right)$ and $B_{n}(t)\left(n=N_{V}, N_{V}+1, \cdots, N_{V}+N_{S}\right)$ are piecewise sinusoidal basis functions:

$$
B_{n}(\tau)= \begin{cases}\frac{\sin \left(\tau-\tau_{n-1}\right)}{\sin \left(\tau_{n}-\tau_{n-1}\right)}, & \tau_{n-1} \leq \tau \leq \tau_{n} \\ \frac{\sin \left(\tau_{n+1}-\tau\right)}{\sin \left(\tau_{n+1}-\tau_{n}\right)}, & \tau_{n} \leq \tau \leq \tau_{n+1} \\ 0, & \text { otherwise }\end{cases}
$$

where the variable $\tau$ denotes $z$ or $t$.

Using the condition $I(l)=0$ at the arm-end point $S_{e}$, we obtain $I_{N_{V}+N_{S}}=0$. Thus, substituting (37) into (34) and choosing

$$
T_{C}\left(l^{\prime}\right)= \begin{cases}B_{m}\left(z^{\prime}\right) & \left(m=0,1, \cdots, N_{V}\right) \\ & \left(0 \leq z^{\prime} \leq h\right) \quad l^{\prime} \in V \\ B_{m}\left(t^{\prime}\right) \quad\left(m=N_{V}, N_{V}+1, \cdots\right. & \\ & \left.N_{V}+N_{S}-1\right) \\ & \left(h \leq t^{\prime} \leq T\right) \quad l^{\prime} \in S\end{cases}
$$

we obtain a set of linear equations

$$
\sum_{n=0}^{N_{V}+N_{S}-1} Z_{m n} I_{n}=V_{m}, \quad \begin{aligned}
& \left(m=0,1, \cdots, N_{V}\right. \\
& \left.N_{V}+1, \cdots, N_{V}+N_{S}-1\right)
\end{aligned}
$$

where

$$
V_{m}=\int_{0}^{h} \phi_{V}\left(z^{\prime}\right) B_{m}\left(z^{\prime}\right) d z^{\prime}
$$


$Z_{m n}$ consists of $Z_{m n}^{V}$ and $Z_{m n}^{S}$, which are given as

$$
\begin{aligned}
Z_{m n}^{V}= & \int_{0}^{h} \int_{h}^{T} B_{m}^{\prime}\left(t^{\prime}\right) B_{n}(z) \frac{\partial}{\partial z}\left[\tilde{G}_{S}^{z}\left(\rho_{v}, z\right)\right] d t^{\prime} d z \\
& +\int_{0}^{h} \int_{0}^{h}\left\{\varepsilon_{r} k_{0}^{2} G_{V}^{z}\left(z, z^{\prime}\right) B_{m}\left(z^{\prime}\right) B_{n}(z)\right. \\
& \left.+B_{m}^{\prime}\left(z^{\prime}\right) B_{n}(z) \frac{\partial}{\partial z}\left[\tilde{G}_{V}^{z}\left(z, z^{\prime}\right)\right]\right\} d z^{\prime} d z
\end{aligned}
$$

and

$$
\begin{aligned}
Z_{m n}^{S}= & \int_{h}^{T} \int_{h}^{T}\left\{k_{0}^{2} G_{s, S}^{s}(\rho) \Gamma\left(t, t^{\prime}\right) B_{m}\left(t^{\prime}\right) B_{n}(t)\right. \\
& \left.+B_{m}^{\prime}\left(t^{\prime}\right) B_{n}(t) \frac{\partial}{\partial t}\left[G_{s, S}^{s}(\rho)+\tilde{G}_{z, S}^{s}(\rho)\right]\right\} d t^{\prime} d t \\
& +\int_{h}^{T} \int_{0}^{h} B_{m}^{\prime}\left(z^{\prime}\right) B_{n}(t) \frac{\partial}{\partial t}\left[\tilde{G}_{S}^{z}\left(\rho, z^{\prime}\right)\right] d z^{\prime} d t \\
& -\int_{h}^{T} B_{m}(h) B_{n}(t) \frac{\partial}{\partial t}\left\{\tilde{G}_{S}^{z}(\rho, h)\right. \\
& \left.-\left[G_{s, S}^{s}(\rho)+\tilde{G}_{z, S}^{s}(\rho)\right]\right\}\left.\right|_{t^{\prime}=h} d t
\end{aligned}
$$

with

$$
\Gamma\left(t, t^{\prime}\right) \equiv\left[\hat{s} \cdot \hat{s}^{\prime}\right] \frac{d s}{d t} \frac{d s^{\prime}}{d t^{\prime}}=x^{\prime}(t) x^{\prime}\left(t^{\prime}\right)+y^{\prime}(t) y^{\prime}\left(t^{\prime}\right)
$$

in which

$$
x^{\prime}(t)=d x(t) / d t, x^{\prime}\left(t^{\prime}\right)=d x\left(t^{\prime}\right) / d t^{\prime}
$$

$y^{\prime}(t)$ and $y^{\prime}\left(t^{\prime}\right)$ are similarly defined. Integrating by parts, we have $Z_{m n}^{V}$ and $Z_{m n}^{S}$

$$
\begin{aligned}
Z_{m n}^{V}= & \int_{h}^{T} B_{m}^{\prime}\left(t^{\prime}\right)\left[B_{n}(h) \tilde{G}_{S}^{z}\left(\rho_{v}, h\right)-B_{n}(0) \tilde{G}_{S}^{z}\left(\rho_{v}, 0\right)\right] d t^{\prime} \\
& -\int_{0}^{h} \int_{h}^{T} B_{m}^{\prime}\left(t^{\prime}\right) B_{n}^{\prime}(z) \tilde{G}_{S}^{z}\left(\rho_{v}, z\right) d t^{\prime} d z \\
& +\int_{0}^{h} B_{m}^{\prime}\left(z^{\prime}\right)\left[B_{n}(h) \tilde{G}_{V}^{z}\left(h, z^{\prime}\right)\right. \\
& \left.-B_{n}(0) \tilde{G}_{V}^{z}\left(0, z^{\prime}\right)\right] d z^{\prime} \\
& +\int_{0}^{h} \int_{0}^{h}\left\{\varepsilon_{r} k_{0}^{2} G_{V}^{z}\left(z, z^{\prime}\right) B_{m}\left(z^{\prime}\right) B_{n}(z)\right. \\
& \left.-B_{m}^{\prime}\left(z^{\prime}\right) B_{n}^{\prime}(z) \tilde{G}_{V}^{z}\left(z, z^{\prime}\right)\right\} d z^{\prime} d z
\end{aligned}
$$

$$
\begin{aligned}
Z_{m n}^{S}= & \int_{h}^{T} B_{m}^{\prime}\left(t^{\prime}\right)\left\{B_{n}(T)\left[G_{s, S}^{s}(\rho)+\tilde{G}_{z, S}^{s}(\rho)\right]_{t=T}\right. \\
& \left.-B_{n}(h)\left[G_{s, S}^{s}(\rho)+\tilde{G}_{z, S}^{s}(\rho)\right]_{t=h}\right\} d t^{\prime} \\
& +\int_{h}^{T} \int_{h}^{T}\left\{k_{0}^{2} G_{s, S}^{s}(\rho) \Gamma\left(t, t^{\prime}\right) B_{m}\left(t^{\prime}\right) B_{n}(t)\right. \\
& \left.-B_{m}^{\prime}\left(t^{\prime}\right) B_{n}^{\prime}(t)\left[G_{s, S}^{s}(\rho)+\tilde{G}_{z, S}^{s}(\rho)\right]\right\} d t^{\prime} d t
\end{aligned}
$$

$$
\begin{aligned}
& +\int_{0}^{h} B_{m}^{\prime}\left(z^{\prime}\right)\left[B_{n}(T) \tilde{G}_{S}^{z}\left(\left.\rho\right|_{t=T}, z^{\prime}\right)\right. \\
& \left.-B_{n}(h) \tilde{G}_{S}^{z}\left(\left.\rho\right|_{t=h}, z^{\prime}\right)\right] d z^{\prime} \\
& -\int_{h}^{T} \int_{0}^{h} B_{m}^{\prime}\left(z^{\prime}\right) B_{n}^{\prime}(t) \tilde{G}_{S}^{z}\left(\rho, z^{\prime}\right) d z^{\prime} d t \\
& -B_{m}(h)\left\{B _ { n } ( T ) \left[\tilde{G}_{S}^{z}(\rho, h)\right.\right. \\
& \left.-\left[G_{s, S}^{s}(\rho)+\tilde{G}_{z, S}^{s}(\rho)\right]\right]_{\substack{t^{\prime}=h \\
t=T}} \\
& \left.-B_{n}(h)\left[\tilde{G}_{S}^{z}(\rho, h)-\left[G_{s, S}^{s}(\rho)+\tilde{G}_{z, S}^{s}(\rho)\right]\right]_{\begin{array}{c}
t^{\prime}=h \\
t=h
\end{array}}\right\} \\
& +\int_{h}^{T} B_{m}(h) B_{n}^{\prime}(t)\left\{\tilde{G}_{S}^{z}(\rho, h)\right. \\
& \left.-\left[G_{s, S}^{s}(\rho)+\tilde{G}_{z, S}^{s}(\rho)\right]\right\}_{t^{\prime}=h} d t .
\end{aligned}
$$

After substituting $\tilde{G}_{S}^{z}\left(\rho_{v}, 0\right)=0$ and $\tilde{G}_{V}^{z}\left(0, z^{\prime}\right)=0$ into (45) and $B_{n}(T)=0$ into (46), we combine $Z_{m n}^{V}$ with $Z_{m n}^{S}$ to obtain $Z_{m n}$

$$
\begin{aligned}
& Z_{m n}= \int_{0}^{h} \int_{0}^{h}\left\{\varepsilon_{r} k_{0}^{2} G_{V}^{z}\left(z, z^{\prime}\right) B_{m}\left(z^{\prime}\right) B_{n}(z)\right. \\
&\left.-B_{m}^{\prime}\left(z^{\prime}\right) B_{n}^{\prime}(z) \tilde{G}_{V}^{z}\left(z, z^{\prime}\right)\right\} d z^{\prime} d z \\
&+\int_{h}^{T} \int_{h}^{T}\left\{k_{0}^{2} G_{s, S}^{s}(\rho) \Gamma\left(t, t^{\prime}\right) B_{m}\left(t^{\prime}\right) B_{n}(t)\right. \\
&\left.-B_{m}^{\prime}\left(t^{\prime}\right) B_{n}^{\prime}(t)\left[G_{s, S}^{s}(\rho)+\tilde{G}_{z, S}^{s}(\rho)\right]\right\} d t^{\prime} d t \\
&-\int_{0}^{h} \int_{h}^{T} B_{m}^{\prime}\left(t^{\prime}\right) B_{n}^{\prime}(z) \tilde{G}_{S}^{z}\left(\rho_{v}, z\right) d t^{\prime} d z \\
&-\int_{h}^{T} \int_{0}^{h} B_{m}^{\prime}\left(z^{\prime}\right) B_{n}^{\prime}(t) \tilde{G}_{S}^{z}\left(\rho, z^{\prime}\right) d z^{\prime} d t \\
&+\int_{h}^{T} B_{m}\left(t^{\prime}\right) B_{n}(h)\left\{\tilde{G}_{S}^{z}\left(\rho_{v}, h\right)\right. \\
&\left.-\left[G_{s, S}^{s}(\rho)+\tilde{G}_{z, S}^{s}(\rho)\right]\right\}_{t=h} d t^{\prime} \\
&+\int_{h}^{T} B_{m}(h) B_{n}^{\prime}(t)\left\{\tilde{G}_{S}^{z}(\rho, h)\right. \\
&\left.-\left[G_{s, S}^{s}(\rho)+\tilde{G}_{z, S}^{s}(\rho)\right]\right\}_{t^{\prime}=h} d t \\
&-B_{m}(h) B_{n}(h)\left[\tilde{G}_{S}^{z}(\rho, h)\right. \\
&\left.-\left[G_{s, S}^{s}(\rho)+\tilde{G}_{z, S}^{s}(\rho)\right]\right]_{t^{\prime}=h} \cdot \\
& t=h
\end{aligned}
$$

Checking (47) term by term, we find that the evaluation of $Z_{m n}$ may be carried out by directly using any of the known numerical integration schemes. This is because all of the integrals in $Z_{m n}$ are definite integrals and all the integrands are a function of either parameter $t$ or variable $z$.

We should note from (41) and (47) that the solution to the current distribution involves the evaluation of six Sommerfeld integrals. The numerical considerations and treatments of the Sommerfeld integration have been presented by various authors [1], [13]-[15]. Two treatments are of particular interest here: A) the first is to alleviate the difficulty in the evaluation of the integrals as source and observation points come close together and B) the second treatment is to save computer 
time in filling of the MoM matrix. In A), the integrals are decomposed with the help of image theory into two parts: 1) the perturbation term (characterized by the presence of surface wave poles), which is a well-behaved integral and 2) the singularity-containing term, which can be treated analytically [10]. For B), we employ the so-called interpolation algorithm [4] in which the Sommerfeld integrals are precomputed on a grid of points in the solution domain and then used repeatedly in a table look-up together with interpolation. For this paper, two one-dimensional (1-D) interpolations in $\rho\left[G_{s_{2} S}^{s}(\rho)\right.$ and $\left.\tilde{G}_{z, S}^{s}(\rho)\right]$, three 1-D interpolations in $z\left[G_{V}^{z}\left(z, z^{\prime}\right), G_{V}^{z}\left(z, z^{\prime}\right)\right.$, and $\phi_{V}(z)$ ], and one two-dimensional (2-D) interpolation in $(\rho, z)\left[\tilde{G}_{S}^{z}(\rho, z)\right]$ are required. Note that $G_{V}^{z}\left(z, z^{\prime}\right)$ can be converted into a combination of two 1-D Sommerfeld integrals [10]. Similarly, $\tilde{G}_{V}^{z}\left(z, z^{\prime}\right)$ can be treated by using the same 1-D Sommerfeld integrals.

\section{Radiation Pattern}

Based on the current distribution solved, the far-zone radiation fields are obtained by using the stationary phase integration approach [10], [16]. Without going into detail, we express the far-zone electric field generated by the vertical probe and printed antenna in the spherical coordinate system $(R, \theta, \phi)$ (see Fig. 1) as

$$
\begin{aligned}
E_{\theta}(\theta, \phi)= & -j 30 k_{0} \frac{e^{-j k_{0} R}}{R} \\
& \cdot\left[S_{\theta}(\theta, \phi) P_{\theta}(\theta)+V_{\theta}(\theta) Q_{\theta}(\theta)\right] \\
E_{\phi}(\theta, \phi)= & -j 30 k_{0} \frac{e^{-j k_{0} R}}{R}\left[S_{\phi}(\theta, \phi) P_{\phi}(\theta)\right]
\end{aligned}
$$

where

$$
\begin{aligned}
S_{\theta}(\theta, \phi)= & \int_{h}^{T}\left[\cos \theta \cos \phi x^{\prime}(t)+\cos \theta \sin \phi y^{\prime}(t)\right] \\
& \cdot I_{S}(t) e^{j k_{0} \Theta(t)} d t \\
S_{\phi}(\theta, \phi)= & \int_{h}^{T}\left[-\sin \phi x^{\prime}(t)+\cos \phi y^{\prime}(t)\right] \\
& \cdot I_{S}(t) e^{j k_{0} \Theta(t)} d t \\
V_{\theta}(\theta)= & -\int_{0}^{h} \sin \theta \cos \left(k_{0} \gamma z\right) I_{V}(z) d z \\
P_{\theta}(\theta)= & \frac{2 \sin \left(k_{0} \gamma h\right)}{\cos \theta \sin \left(k_{0} \gamma h\right)-j \gamma \cos \left(k_{0} \gamma h\right)} \\
& \cdot \frac{\gamma\left[\gamma \cos \left(k_{0} \gamma h\right)+j \cos \theta \sin \left(k_{0} \gamma h\right)\right]}{\varepsilon_{r} \cos \left(k_{0} \gamma h\right) \cos \theta+j \gamma \sin \left(k_{0} \gamma h\right)} \\
P_{\phi}(\theta)= & \frac{2 \cos \theta \sin \left(k_{0} \gamma h\right)}{\cos \theta \sin \left(k_{0} \gamma h\right)-j \gamma \cos \left(k_{0} \gamma h\right)} \\
Q_{\theta}(\theta)= & \frac{2 \cos \theta}{\varepsilon_{r} \cos \left(k_{0} \gamma h\right) \cos \theta+j \gamma \sin \left(k_{0} \gamma h\right)} \\
& \cdot e^{j k_{0} h \cos \theta}
\end{aligned}
$$

with

$$
\begin{aligned}
\Theta(t) & =x(t) \sin \theta \cos \phi+y(t) \sin \theta \sin \phi+h \cos \theta \\
\gamma & =\sqrt{\varepsilon_{r}-\sin ^{2} \theta} .
\end{aligned}
$$

Note that the integrals in (50)-(52) can be evaluated directly by using a numerical integration scheme.

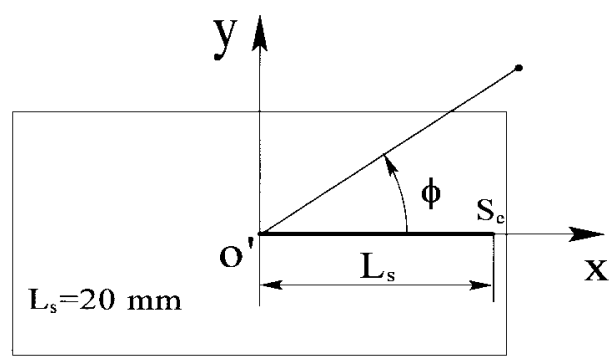

(a)

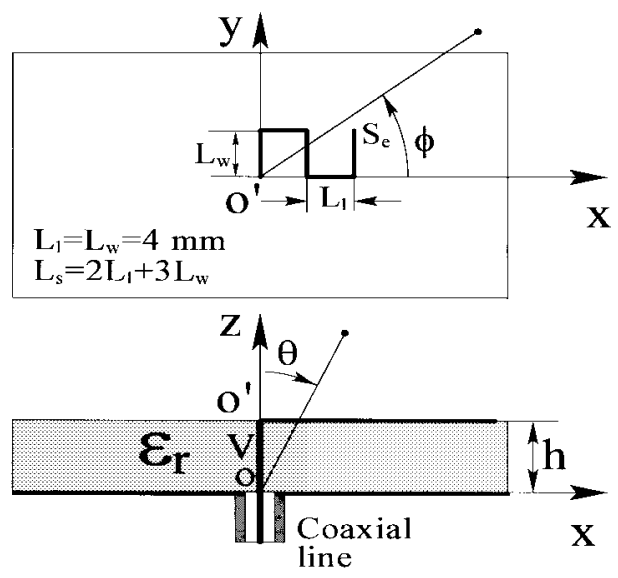

(b)

Fig. 2. Configurations of (a) a printed linear antenna and (b) a printed meander antenna.

\section{NUMERICAL EXAMPLES}

\section{A. Linear and Meander Antennas}

To examine the accuracy of the computer program developed by using the previous formulation, a straight linear antenna and a meander antenna are first analyzed. The two antennas (shown in Fig. 2) have the same printed wire length $L_{s}=20 \mathrm{~mm}$ and the same wire radius $a_{s}=0.1 \mathrm{~mm}$. Both are printed on a grounded dielectric substrate with a thickness of $h=6.35 \mathrm{~mm}$ and a dielectric constant of $\varepsilon_{r}=2.45$. The printed antennas are excited by a vertical probe from a coaxial line with inner and outer radii of $a_{v}=0.635$ and $b=2.065$ mm, respectively.

The input impedance computed for the linear and meander antennas is shown in Fig. 3 with the experimental data. We can see good agreement between the computed and experimental results.

The current distributions at a frequency of $3.0 \mathrm{GHz}$ are depicted in Fig. 4, where the arm length is normalized to the total wire length $L=L_{s}+h$. Fig. 5 presents a comparison between the computed and measured results for the radiation patterns at 3.0 GHz. We observe that the measured and computed results are in agreement, but the discrepancy between the results near $\theta= \pm 90^{\circ}$ is large. This is due to the stationary phase method used in the development of the formula for the radiation fields, which is not valid when $\theta= \pm 90^{\circ}$.

\section{B. Circular Open Loop}

A circular closed-loop antenna excited by a center-fed source radiates a linearly polarized wave [6]. A recent study 


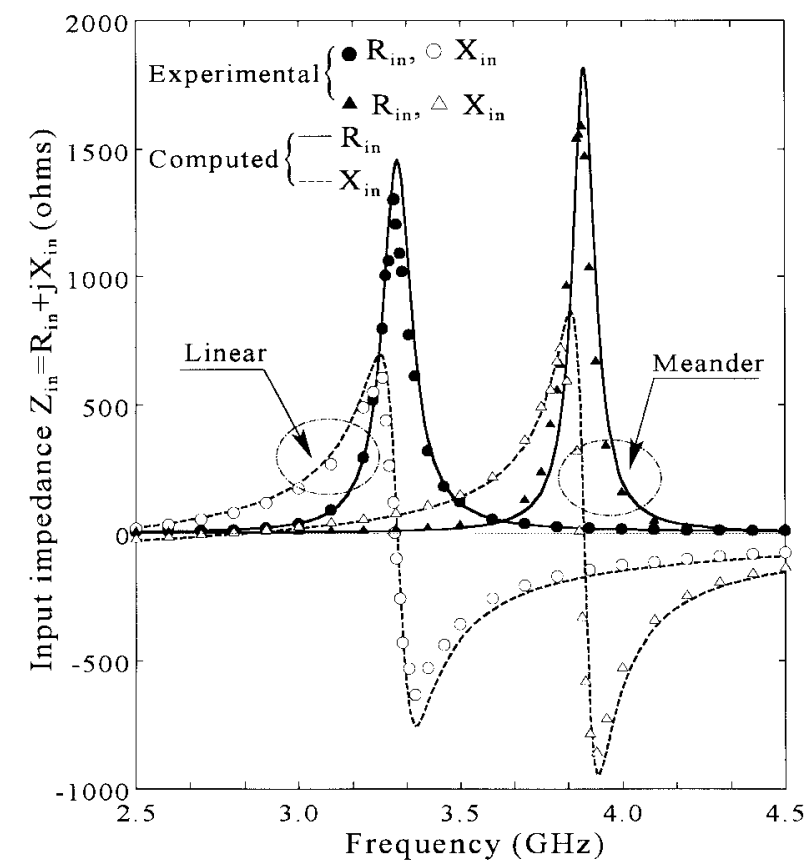

Fig. 3. Input impedance of linear and meander antennas versus frequency.

has shown that a loop with a small gap in free-space can radiate a circularly polarized wave [17].

Let us consider a printed circular loop antenna with a gap of $\phi_{g}=15^{\circ}$, which is excited by a vertical probe, as shown in Fig. 6. The computed frequency responses of the input impedance and axial ratio along the $+z$ axis are shown in Fig. 7. The frequency bandwidth where the axial ratio is less than $3 \mathrm{~dB}$ is approximately $10 \%$. We also observe that over this frequency bandwidth the antenna shows a desirable input impedance characteristic. The axial ratio and input impedance at a design frequency of $f_{0}$ are found to be about $0.15 \mathrm{~dB}$ and $(65-j 20) \Omega$, respectively. The current distribution at $f_{0}$ is presented in Fig. 8. Note that the arm length is normalized to a total arm length (including the vertical probe length) of $1.0231 \lambda_{0}$. The smooth decay of current amplitude along the arm shows that an open loop can operate as a traveling wave antenna with minimal reflections from the arm end. Fig. 9 shows a circular polarization radiation pattern at $f_{0}$ in which $E_{R}$ and $E_{L}$ denote the right-hand and left-hand circularly polarized wave components, respectively.

\section{Archimedian Spiral}

It has been shown that a monofilar spiral antenna in freespace [18] can radiate a tilted circularly polarized beam. This antenna is applied to land-based mobile communication systems. Using the previous formulation, we consider the radiation properties of a printed Archimedian spiral antenna defined by the function $r=A \phi$ ( $A$ is the spiral constant and $\phi$ is the winding angle starting at $\phi_{s}$ ). The configuration and pertinent parameters of the antenna are depicted in Fig. 10.

It is found that the maximum radiation occurs in a direction of $(\theta, \phi)=\left(33^{\circ}, 335^{\circ}\right)$. The calculated axial ratio and gain in the maximum radiation direction, as well as the input impedance are shown as a function of the arm length in

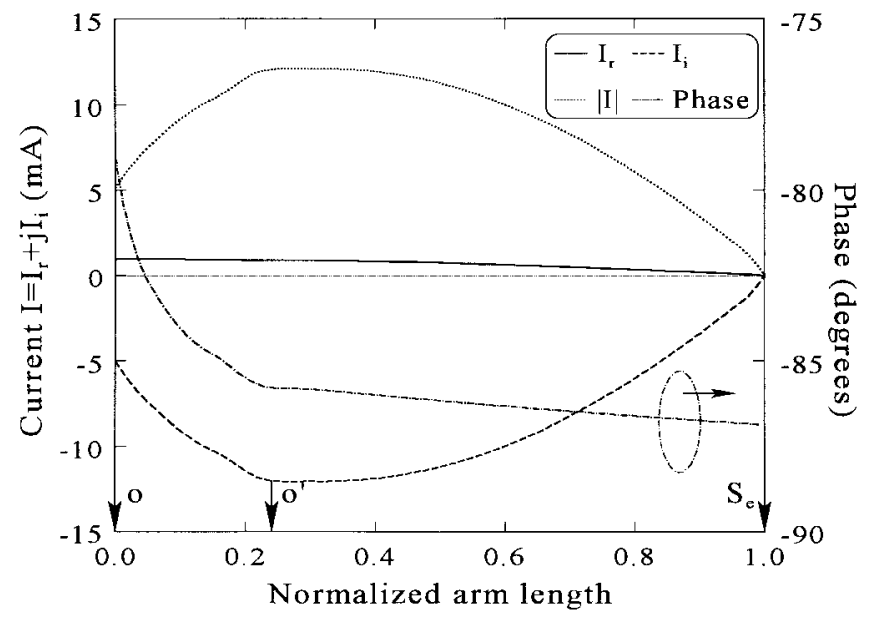

(a)

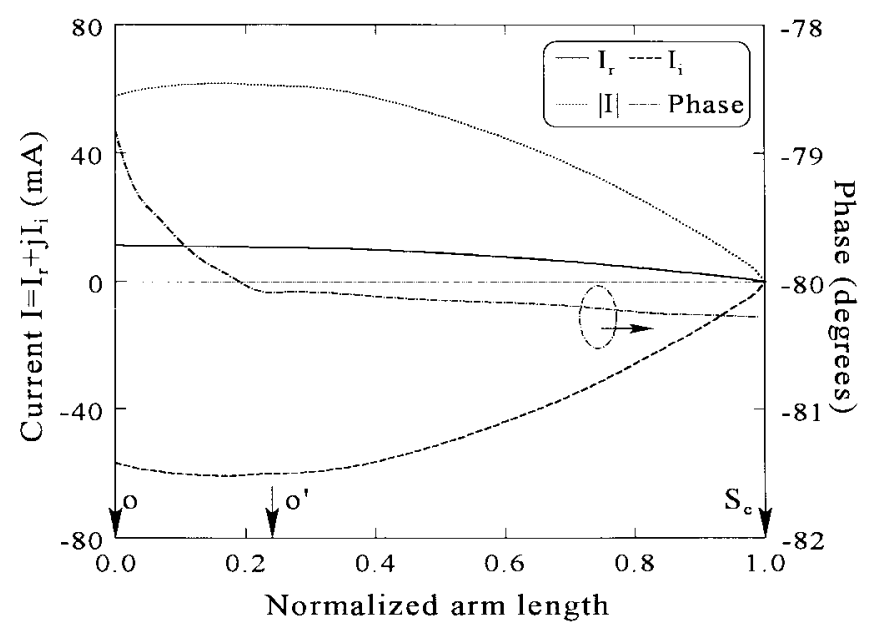

(b)

Fig. 4. Current distributions at $3 \mathrm{GHz}$ along (a) a linear antenna and (b) a meander antenna.

Fig. 11, where the arm length is measured from point $o$ to the arm end $S_{e}$. It is observed that the antenna has an axial ratio of less than $3 \mathrm{~dB}$ over a wide range of arm lengths (from $L_{1}=3.3 \lambda_{0}$ to $L_{2}=4.9 \lambda_{0}$ ) while the gain and input impedance remain relatively constant. This implies that the spiral antenna has a wide bandwidth performance. The current distribution for an arm length of $L_{m}=4.13 \lambda_{0}$, which corresponds to a minimal axial ratio of $0.53 \mathrm{~dB}$, is shown in Fig. 12. The radiation pattern for $L_{m}=4.13 \lambda_{0}$ is presented in Fig. 13, which confirms that the beam is tilted.

\section{CONCLUSIONS}

A general numerical technique based on an integral equation for antenna current is developed for analysis of an arbitrarily shaped probe-excited single-arm printed wire antenna. A unified current integral equation is formulated in conjunction with the dyadic Green's functions and reciprocity theorem. The current distribution along the printed wire and feed probe is determined by using the parametric moment method, which results in an exact representation of printed wire configurations and a more efficient computational formulation. The farzone electric field from the printed wire and feed probe is 
Computed $\left\{\begin{array}{l}-\mathrm{E}_{\theta} \\ -\mathrm{E}_{\phi}\end{array}\right.$ Experimental $\left\{\begin{array}{l}\bullet \mathrm{E}_{\theta} \\ \mathrm{E}_{\phi}\end{array}\right.$
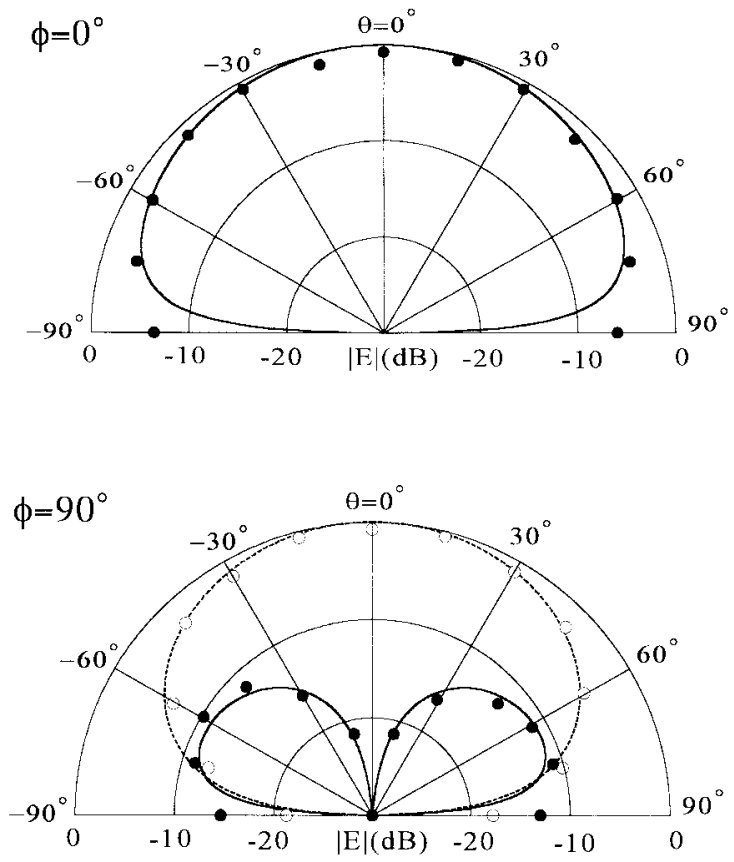

(a)
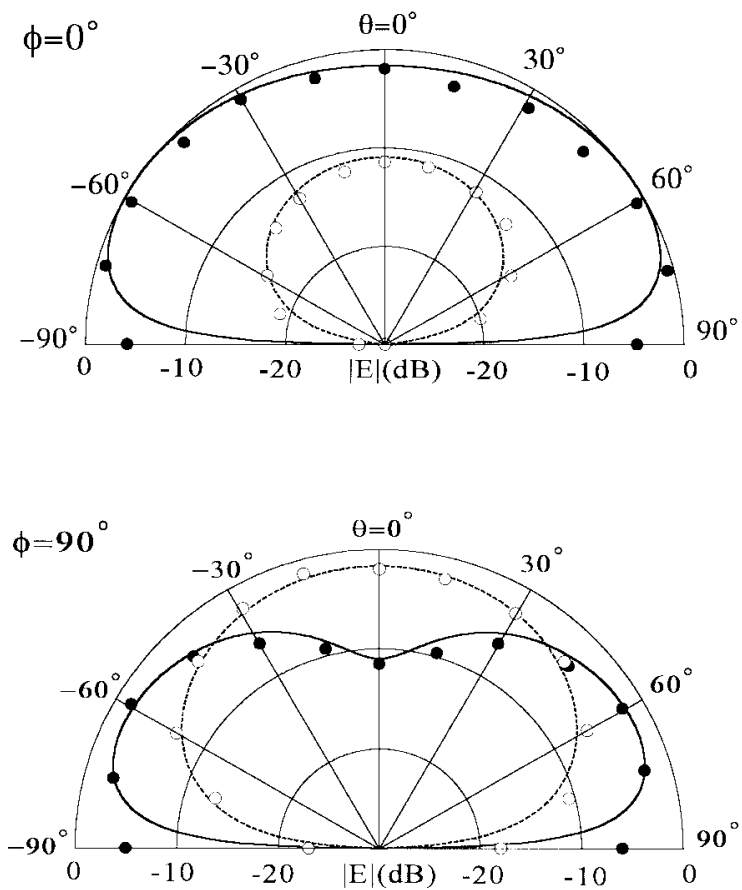

(b)

Fig. 5. Radiation patterns at $3 \mathrm{GHz}$ of (a) a linear antenna and (b) a meander antenna.

also presented. Good agreement between the computed and experimental results of the input impedance and radiation patterns for linear and meander antennas is obtained, verifying the validity of the formulation. To show the applicability of this technique to printed antennas with vertical probe feed, we also investigate a circular open loop and an Archimedian

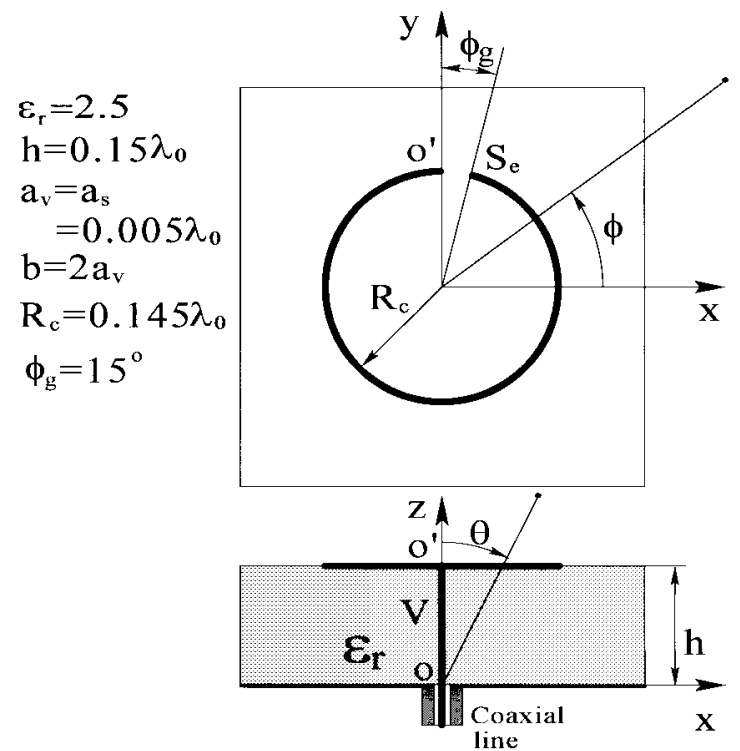

Fig. 6. Geometry and parameters of a printed circular open-loop antenna excited by a vertical probe.

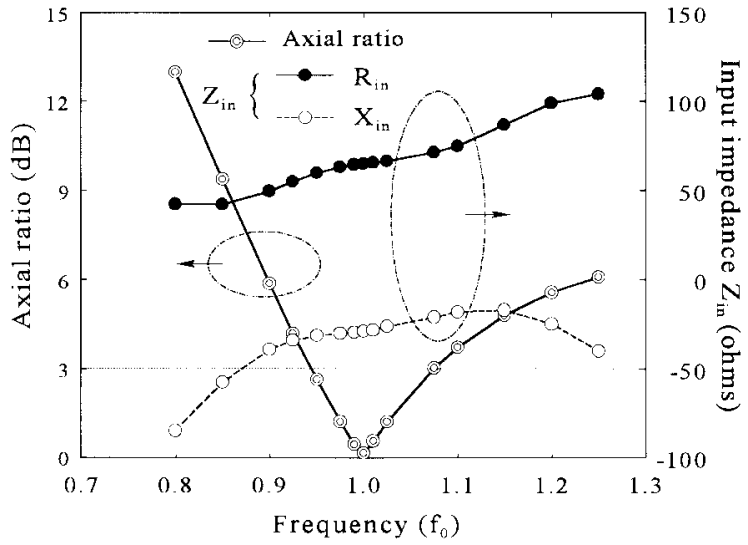

Fig. 7. Frequency responses of the axial ratio and input impedance for a printed circular open-loop antenna versus frequency.

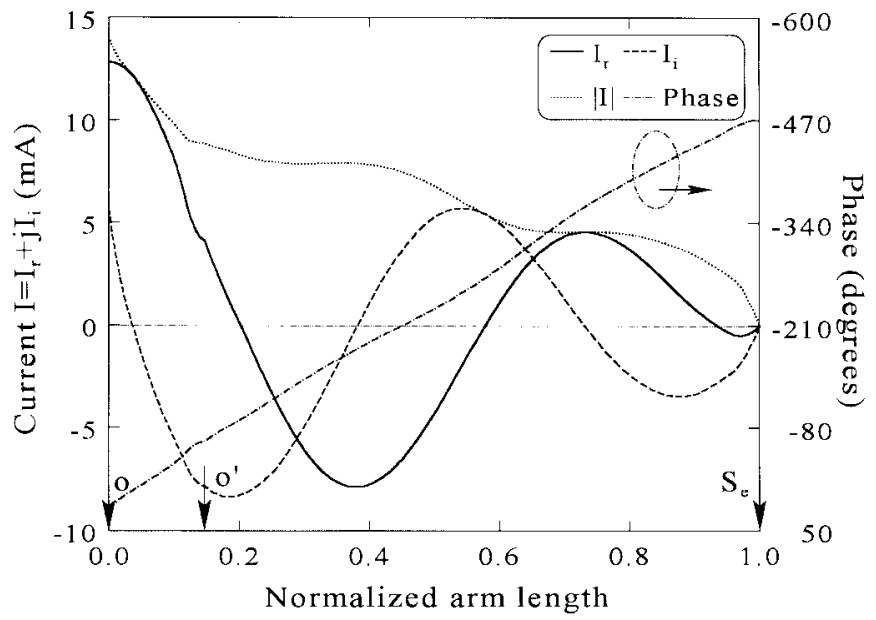

Fig. 8. Current distribution along a printed circular open-loop antenna at $f_{0}$. 

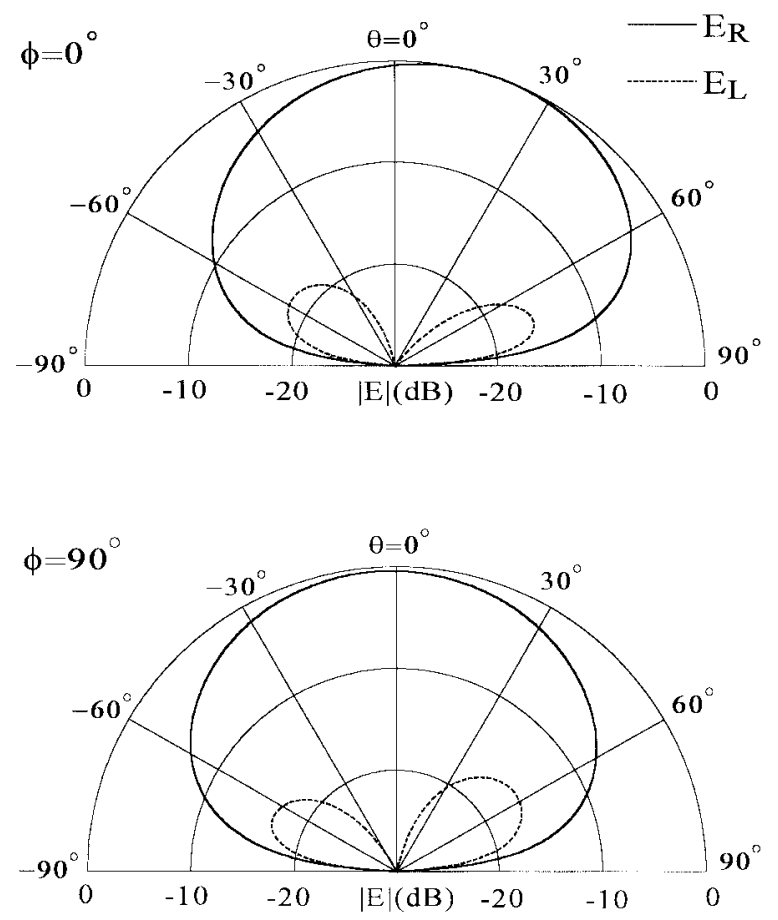

Fig. 9. Radiation pattern of a printed circular open-loop antenna at $f_{0}$.

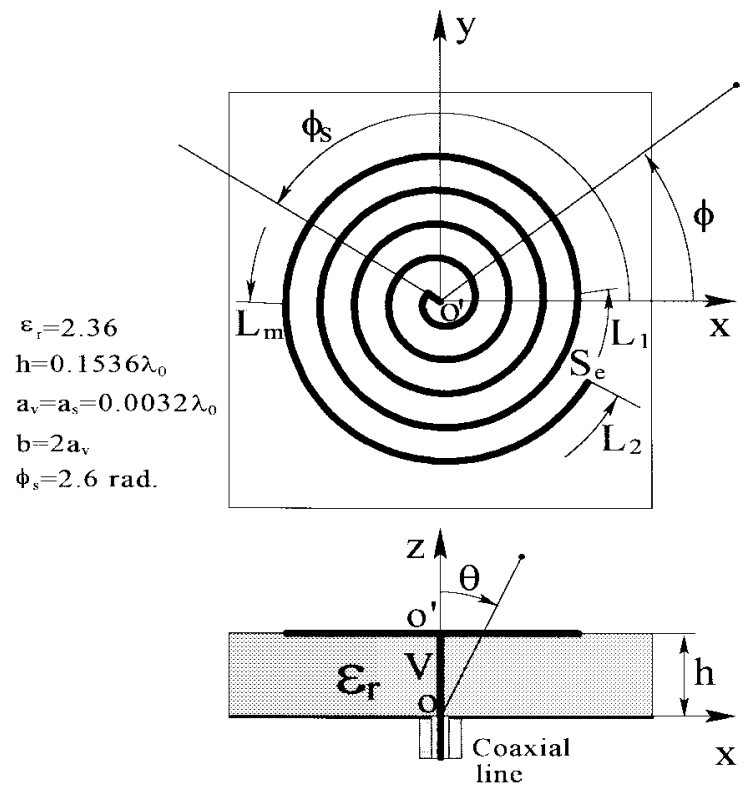

Fig. 10. Configuration and parameters of a printed Archimedian spiral antenna with a spiral constant of $A=0.01 \lambda_{0} / \mathrm{rad}$.

spiral. It is found that both can serve as radiation elements of circular polarization.

\section{APPENDIX \\ PROOF OF $\cos \Phi=-\left(\partial \rho / \partial s^{\prime}\right)$}

In a coordinate system measured with the arc length along the wire, the distance $\rho$ between an observation point $(\vec{r})$ and a source point $\left(\vec{r}^{\prime}\right)$ is expressed as $\rho=\rho\left(s, s^{\prime}\right)$, where $s(\vec{r})$ and $s^{\prime}\left(\vec{r}^{\prime}\right)$ denote the arc length coordinates, as shown in Fig. 14.

Let the source point change a little from $\vec{r}^{\prime}$ to $\vec{r}^{\prime}+\Delta \vec{r}^{\prime}$, where $\Delta \vec{r}^{\prime}$ is an increment of the position vector $\vec{r}^{\prime}$. The

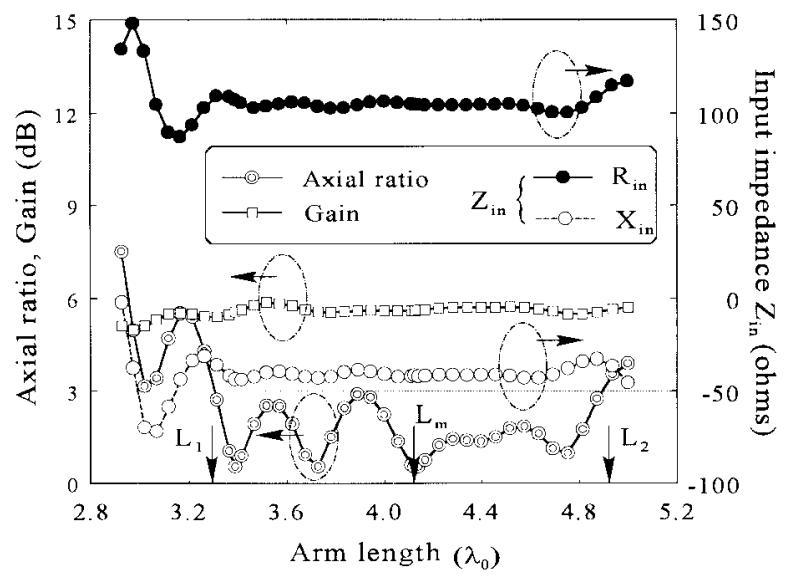

Fig. 11. Axial ratio, gain, and input impedance as a function of the arm length for a printed Archimedian spiral antenna.

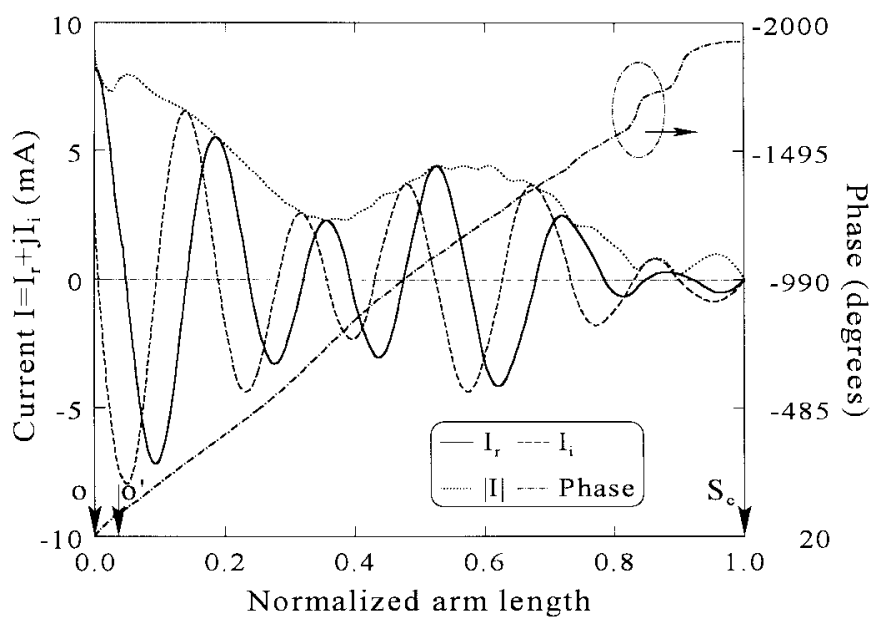

Fig. 12. Current distribution along a printed Archimedian spiral antenna with an arm length of $L_{m}=4.13 \lambda_{0}$.

arc length coordinate at the point of $\vec{r}^{\prime}+\Delta \vec{r}^{\prime}$ should be $s^{\prime}+\Delta s^{\prime}$, where $\Delta s^{\prime}$ is the arc length between $\vec{r}^{\prime}$ and $\vec{r}^{\prime}+\Delta \vec{r}^{\prime}$. Therefore, the distance between the two points of $\bar{r}$ and $\bar{r}^{\prime}+\Delta \bar{r}^{\prime}$ becomes $\rho=\rho\left(s, s^{\prime}+\Delta s^{\prime}\right)$.

From Fig. 14, we can directly obtain that

$$
\cos \Phi^{\prime}=\frac{\rho\left(s, s^{\prime}\right)-\rho\left(s, s^{\prime}+\Delta s^{\prime}\right) \cos \alpha}{\Delta r^{\prime}} .
$$

Note that (58) holds true even for an obtuse angle $\Phi^{\prime}$.

Since $\lim _{\Delta r^{\prime} \rightarrow 0} \Phi^{\prime}=\Phi, \lim _{\Delta r^{\prime} \rightarrow 0} \alpha=0$, and $\lim _{\Delta r^{\prime} \rightarrow 0} \Delta r^{\prime}=\Delta s^{\prime}$, we have

$$
\begin{aligned}
\cos \Phi & =\lim _{\Delta r^{\prime} \rightarrow 0} \cos \Phi^{\prime} \\
& =\lim _{\Delta r^{\prime} \rightarrow 0} \frac{\rho\left(s, s^{\prime}\right)-\rho\left(s, s^{\prime}+\Delta s^{\prime}\right) \cos \alpha}{\Delta r^{\prime}} \\
& =-\lim _{\Delta s^{\prime} \rightarrow 0} \frac{\rho\left(s, s^{\prime}+\Delta s^{\prime}\right)-\rho\left(s, s^{\prime}\right)}{\Delta s^{\prime}}
\end{aligned}
$$




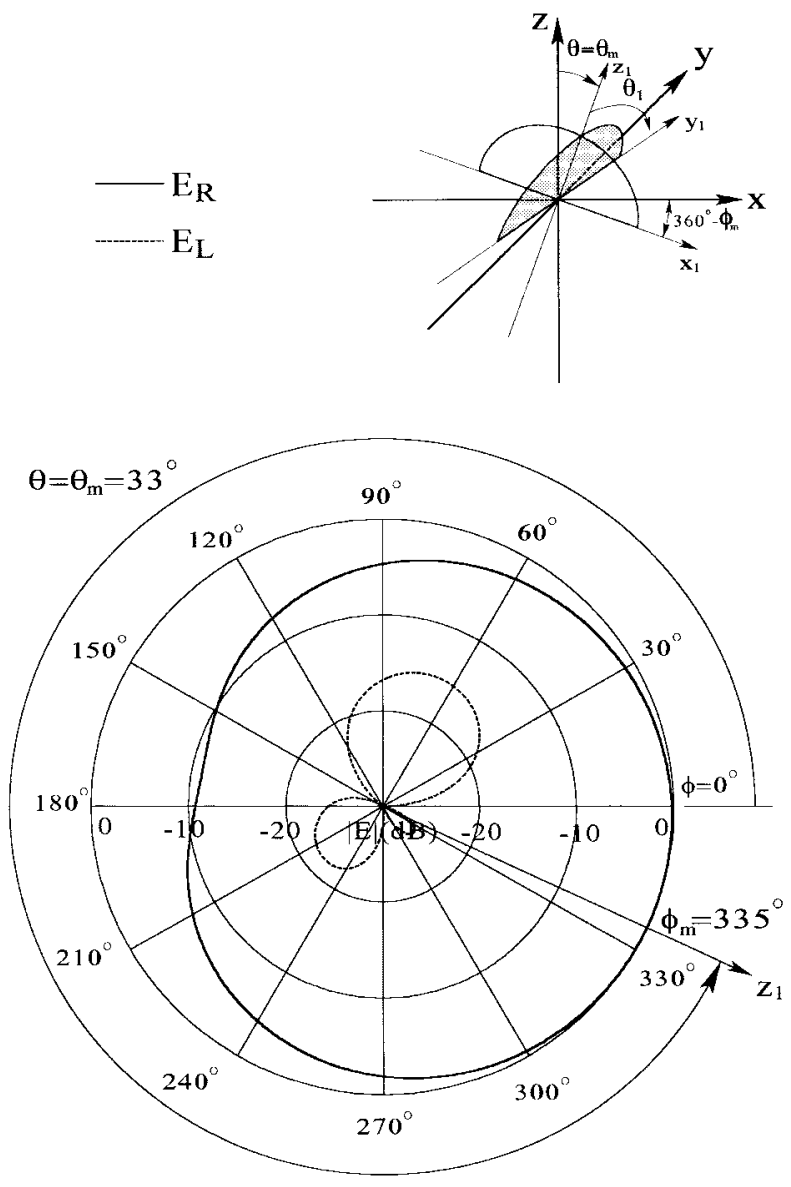

(a)

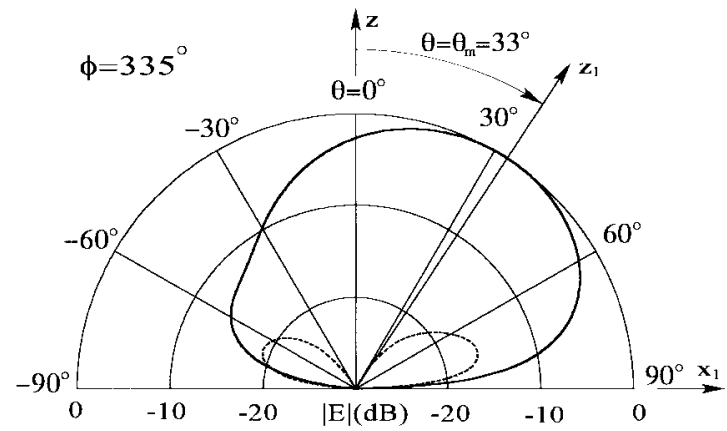

(b)

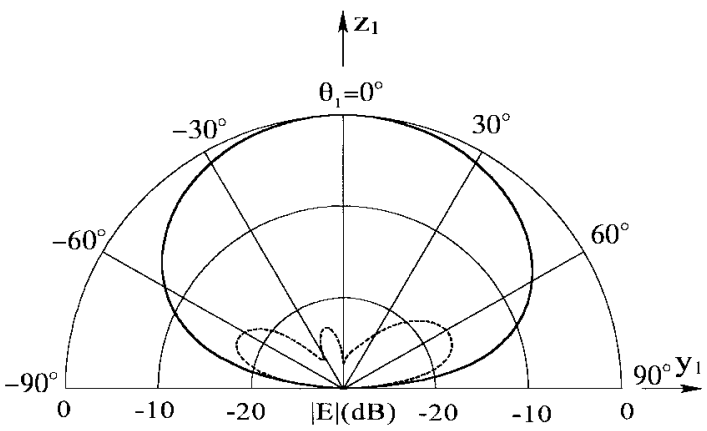

(c)

Fig. 13. Radiation patterns of a printed Archimedian spiral antenna with an arm length of $L_{m}=4.13 \lambda_{0}$. (a) Radiation pattern as a function of azimuth angle $\phi\left(\theta=\theta_{m}=33^{\circ}\right)$. (b) Radiation pattern in the $\phi=\phi_{m}=335^{\circ}$ plane (the $x_{1}-z$ plane in the inset). (c) Radiation pattern in the $y_{1}-z_{1}$ plane (the shaded plane in the inset).

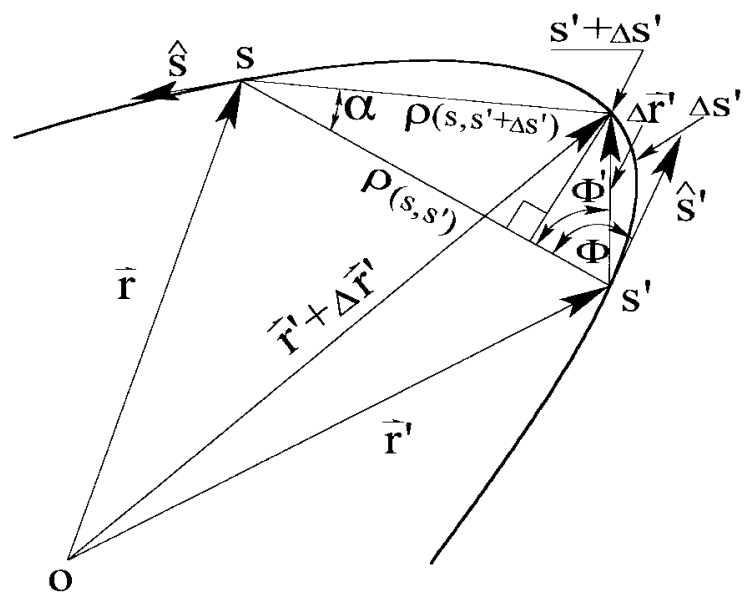

Fig. 14. Proof of $\cos \Phi=-\partial \rho / \partial s^{\prime}$.

which is just the definition of the partial derivative of $-\rho\left(s, s^{\prime}\right)$ with respect to $s^{\prime}$.

\section{REFERENCES}

[1] I. E. Rana and N. G. Alexopoulos, "Current distribution and input impedance of printed dipoles," IEEE Trans. Antennas Propagat., vol. AP-29, pp. 99-105, Jan. 1981.

[2] H. Nakano, S. R. Kerner, and N. G. Alexopoulos, "The moment method solution for printed wire antennas of arbitrary configuration," IEEE Trans. Antennas Propagat., vol. 36, pp. 1667-1674, Dec. 1988.

[3] H. Nakano, K. Hirose, T. Suzuki, S. R. Kerner, and N. G. Alexopoulos, "Numerical analysis of printed line antennas," Proc. Inst. Elect. Eng., vol. 136, pt. H, no. 2, pp. 98-104, Apr. 1989.

[4] H. A. Ragheb and L. Shafai, "Analysis of arbitrary shape printed line microstrip antennas," IEEE Trans. Antennas Propagat., vol. 38, pp. 269-274, Feb. 1990

[5] N. J. Champagne, II, J. T. Williams, and D. R. Wilton, "Analysis of resistively loaded, printed spiral antennas," Electromagn., vol. 14, pp. 363-395, 1994.

[6] H. A. N. Hejase, "Analysis of a printed wire loop antenna," IEEE Trans. Antennas Propagat., vol. 42, pp. 227-233, Feb. 1994.

[7] S. K. Khamas and G. G. Cook, "Moment-method analysis of printed wire spiral using curved piecewise sinusoidal subdomain basis and testing functions," IEEE Trans. Antennas Propagat., vol. 45, pp. 1016-1022, June 1997.

[8] R. C. Johnson, Antenna Engineering Handbook, 3rd ed. New York: McGraw-Hill, 1993, sec. 43-6.

[9] S. K. Khamas, G. G. Cook, R. J. Waldron, and R. M. Edwards, "Moment method analysis of printed single-arm wire spiral antennas using curved segments," Proc. Inst. Elect. Eng., vol. 144, pt. H, no. 4, pp. 261-265, Aug. 1997.

[10] C. L. Chi and N. G. Alexopoulos, "Radiation by a probe through a substrate," IEEE Trans. Antennas Propagat., vol. AP-34, pp. 1080-1091, Sept. 1986

[11] J. R. Mosig and F. E. Gardiol, "Analytical and numerical techniques in Green's function treatment of microstrip antennas," Proc. Inst. Elect. Eng., vol. 130, pt. H, no. 2, pp. 175-182, Mar. 1983.

[12] R. Li, G. Ni, J. Yu, and Z. Jiang, "A new numerical technique for calculating current distributions on curved wire antennas-Parametric B-spline finite element method," IEEE Trans. Magn., vol. 32, no. 3, pp. 906-909, May 1996.

[13] G. Annaert, "Evaluation of Sommerfeld integrals using Chebyshev decomposition," IEEE Trans. Antennas Propagat., vol. 41, pp. 159-164, Feb. 1993.

[14] S. L. Dvorak and E. F. Kuester, "Numerical computation of 2D Sommerfeld integrals-Decomposition of the angular integral," J. Computat. Phys. 98, pp. 199-216, 1992.

[15] _ "Numerical computation of 2-D Sommerfeld integrals-A novel asymptotic extraction technique," J. Computat. Phys. 98, pp. 217-230, 1992.

[16] N. K. Uzunoglu, N. G. Alexopoulos, and J. G. Fikioris, "Radiation properties of microstrip dipoles," IEEE Trans. Antennas Propagat., vol. AP-27, pp. 853-858, Sept. 1979. 
[17] H. Nakano, H. Yoshida, and Y. Wu, "C-figured loop antennas," Inst. Elect. Eng. Electron. Lett., vol. 31, no. 9, pp. 693-694, Apr. 1995.

[18] H. Nakano, Y. Shinma, and J. Yamauchi, "A monofilar spiral antenna and its array above a ground plane-Formulation of a circularly polarized, tilted fan beam," IEEE Trans. Antennas Propagat., vol. 45, pp. 1506-1511, Oct. 1997.

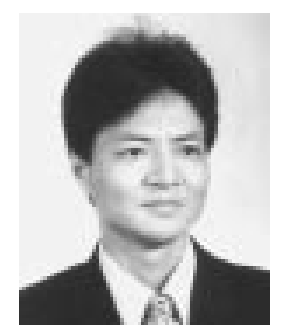

Rong-Lin Li was born in Chongqing, China, on November 8, 1963. He received the B.S. degree in electrical engineering from Xi' an Jiaotong University, Xi' an, China, in 1983, and the M.S. and Ph.D. degrees in electrical engineering from Chongqing University, Chongqing, China, in 1990 and 1994, respectively.

From 1994 to 1996, he was a Postdoctoral Research Fellow at Zhejiang University, Hangzhou, China. From 1997 to 1998, he was an HIF (Hosei International Fund) Research Fellow at Hosei University, Tokyo, Japan. Currently, he is a Professor of the Electrical Engineering Department at Zhejiang University. His research interests include wire antennas, wave propagation in layered media, and computational electromagnetics.

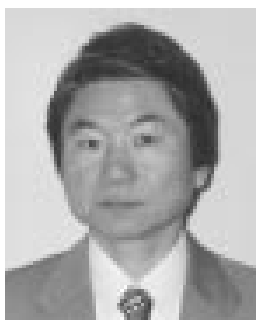

Hisamatsu Nakano (M'75-SM'87-F'92) was born in Ibaraki, Japan, on April 13, 1945. He received the B.E., M.E., and Dr.E. degrees in electrical engineering from Hosei University, Tokyo, in 1968, 1970, and 1974, respectively.

Since 1973 , he has been a member of the faculty of Hosei University, where he is now a Professor in the Electronic Informatics Department. He was a Visiting Associate Professor at Syracuse University (March-September, 1981), a Visiting Professor at University of Manitoba (March-September, 1986), and a Visiting Professor at University of California, Los Angeles (September 1986-March, 1987). He developed a parabolic reflector antenna using a backfire helical feed for direct reception of broadcasting satellite TV programs (DBS) in Japan. He also developed two types of small flat DBS antennas using novel elements: curled and extremely low-profile helical elements. His other developments include microstrip antennas for global positioning systems, wire antennas for portable telephones, and parabolic reflector antennas for reception of communication satellite TV programs. He has published more than 130 refereed journal papers. He is the author of Helical and Spiral Antennas (Letchworth, U.K.: Research Studies Press, 1987) and the coauthor of Analysis Methods of Electromagnetic Wave Problems, Volume 2 (Norwood, MA: Artech House, 1996). His research topics include numerical methods for microwave antennas and light wave problems.

Dr. Nakano is a member of the IEICE in Japan. 